\title{
Nordic Walking Enhances Oxygen Uptake without Increasing the Rate of Perceived Exertion in Patients with Chronic Obstructive Pulmonary Disease
}

\author{
Anael Barberan-Garciaa, b, g Ane Arbillaga-Etxarric, d Elena Gimeno-Santos c, d, e \\ Diego Agustín Rodríguez ${ }^{\mathrm{f} g}$ Yolanda Torralba ${ }^{\mathrm{a}, \mathrm{g}} \quad$ Josep Roca ${ }^{\mathrm{a}, \mathrm{b}, \mathrm{g}} \quad$ Jordi Vilaró ${ }^{\mathrm{C}}$ \\ ${ }^{a}$ Hospital Clínic de Barcelona, Thorax Clinic Institute, Respiratory Diagnostic Center, ${ }^{b}$ Institut d'Investigacions Biomèdiques \\ August Pi i Sunyer (IDIBAPS), University of Barcelona, 'Epidemiology Department, Center of Research in Environmental \\ Epidemiology (CREAL), d Centro de Investigación Biomédica en Red de Epidemiología y Salud Pública (CIBERESP), e Faculty \\ of Health Sciences Blanquerna, Physiotherapy Research Group (GReF), Ramon Llull University, and f Pulmonary Medicine \\ Department, Institut Hospital del Mar d'Investigacions Mèdiques (IMIM), Parc de Salut Mar, Pompeu Fabra University, \\ Barcelona, and ${ }^{9}$ Centro de Investigación Biomédica en Red de Enfermedades Respiratorias (CIBERES), Bunyola, Spain
}

\section{Key Words}

Chronic obstructive pulmonary disease - Exercise physiology · Exercise test · Nordic walking · Physical exertion $\cdot$ Pulmonary rehabilitation

\begin{abstract}
Background: In healthy subjects, Nordic walking (NW) generates higher oxygen uptake $\left(\mathrm{V}_{2}\right)$ than standard walking at an equal rate of perceived exertion (RPE). The feasibility and positive outcomes of NW in patients with chronic obstructive pulmonary disease (COPD) have been reported. Objectives: The aim of the current study is to assess the physiological responses and RPE during NW in COPD patients. Methods: In 15 COPD patients [mean (SD) age 67 (9) years] with a forced expiratory volume in the $1 \mathrm{st} \mathrm{s}$ of $55 \%(15)], \dot{\mathrm{V}}_{2}$, $\mathrm{min}$ ute ventilation and heart rate were measured with a portable system during the 6-min walking test (6MWT), incremental shuttle walking test (SWT), 6-min NW on solid ground $\left(6 \mathrm{mNW}_{\text {ground }}\right)$ and 6-min NW on soft dry beach sand $\left(6 \mathrm{mNW}_{\text {sand }}\right)$. The RPE using a modified Borg scale was assessed after each test. Results: $6 \mathrm{mNW}_{\text {ground }}$ and $6 \mathrm{mNW}_{\text {sand }}$ showed a higher $\dot{\mathrm{V}}_{2}$ plateau compared with the $6 \mathrm{MWT}$ and
\end{abstract}

peak $\dot{\mathrm{V}}_{2}$ measured during SWT [mean (SD) $\dot{\mathrm{V}}_{2} 21$ (3), 22 (4), 18 (4) and 19 (5) $\mathrm{ml}^{\circ} \mathrm{kg}^{-1} \cdot \mathrm{min}^{-1}$, respectively; $\mathrm{p}<0.05$ each]. However, no differences in RPE were observed among $6 \mathrm{mNW}_{\text {ground, }} 6 \mathrm{MWT}$ and SWT [modified Borg scale score for dyspnea 4.2 (2.0), 4.1 (1.8) and 4.3 (1.7), respectively; nonsignificant]. However, RPE in $6 \mathrm{mNW}_{\text {sand }}$ was significantly higher than in all the other exercise protocols [modified Borg scale score for dyspnea 5.2 (2.2); $p<0.05]$. Conclusions: In COPD patients, the use of Nordic poles generates higher $\dot{V}_{2}$ than standard walking with no differences in the dyspnea score. The results indicate the potential to enhance communitybased training programs in these patients.

(c) 2015 S. Karger AG, Basel

\section{Introduction}

Nordic walking (NW) is a walking modality carried out using special poles with rubber or spike tips resembling Nordic style skiing and constitutes a common outdoor physical activity carried out by healthy people worldwide [1]. Moreover, NW has shown effectiveness as a training method to improve aerobic capacity and qual-

\section{KARGER 125}

C 2015 S. Karger AG, Basel

$0025-7931 / 15 / 0893-0221 \$ 39.50 / 0$

E-Mail karger@karger.com

www.karger.com/res
Anael Barberan-Garcia

Hospital Clínic de Barcelona

Thorax Clinic Institute, Respiratory Diagnostic Center

Villarroel 170, ES-08036 Barcelona (Spain)

E-Mail anbarber@ clinic.ub.es 
ity of life both in healthy subjects and patients with chronic conditions [2], including chronic obstructive pulmonary disease (COPD) [3].

The main purpose of NW is to exercise the upper limb and trunk muscles, which are not activated during standard walking. In healthy subjects, this increase in the exercising muscle mass translates into higher oxygen uptake [4] with equal $[5,6]$ or only slightly higher [7] levels of perceived exertion than during standard walking. However, there is no information on the physiological responses during NW in COPD patients in whom ventilatory limitation to exercise might lead to dynamic hyperinflation and a higher rate of perceived exertion (RPE) [8] that could eventually preclude generalization of the findings reported in healthy subjects [4-7].

The rationale behind the current study is that a better knowledge of the physiological responses during $\mathrm{NW}$ in COPD patients may facilitate the design of exercise training programs based on this modality of exercise. In the current research, we assessed the physiological responses during 6 min of NW on two different surfaces that were chosen because they are easily available in many urban environments: solid ground $\left(6 \mathrm{mNW}_{\text {ground }}\right)$ and soft dry beach sand $\left(6 \mathrm{mNW}_{\text {sand }}\right)$. The measurements were compared with those obtained during both the standard 6-min walking test (6MWT) and the incremental shuttle walking test (SWT).

\section{Methods}

\section{Study Design}

Sixteen clinically stable COPD patients (outpatients from the Hospital Clínic de Barcelona) were recruited from February to March 2010 for this study. Inclusion criteria were: (i) a diagnosis of COPD according to the criteria defined by the Global Initiative for Chronic Obstructive Lung Diseases [9]; (ii) no clinical evidence of major cardiovascular or neuromuscular diseases; (iii) no need for supplementary oxygen during exercise, and (iv) tolerance of the facemask during exercise testing. Of note, 10 of the 15 patients were on combined therapy with a long-acting anticholinergic compound and a long-acting $\beta_{2}$-agonist, and the remaining 5 patients on inhaled corticosteroids combined with a long-acting $\beta_{2}$-agonist. Moreover, 4 patients were also treated with simvastatin and 5 patients received furosemide for the treatment of hypercholesterolemia and arterial hypertension, respectively. All patients agreed to participate and signed the informed consent approved by the Ethics Committee for Clinical Research at Hospital Clínic de Barcelona.

For all patients, clinical history was obtained and physical examination was performed. Standard measurements included: (i) forced spirometry and static lung volumes (Master-Screen; Jaeger, Würzburg, Germany); (ii) lung single-breath CO transfer capacity (Master-Screen); (iii) arterial blood gases (partial $\mathrm{O}_{2}$ and $\mathrm{CO}_{2}$ pres- sure in arterial blood) at rest (800 series; Ciba Corning, Medfield, Mass., USA), and (iv) bioelectrical impedance to determine body composition (Quantum X - Bioelectrical Body Composition Analyzer; RJL Systems, Clinton Township, Mich., USA).

\section{Exercise Measurements}

All patients became familiar with the NW technique after being coached by a specialized professional. All of them performed four exercise protocols in two different days in random order: (i) incremental SWT [10] to assess peak oxygen uptake $\left(\dot{\mathrm{V}}_{2}\right)$; (ii) 6MWT [11]; (iii) $6 \mathrm{mNW}_{\text {ground }}$, and (iv) $6 \mathrm{mNW}_{\text {sand }}$. The two NW tests were performed according to the American Thoracic Society guidelines for 6MWT [11] to ensure comparability among exercise protocols.

During all exercise testing, patients breathed through an adult facemask (Hans Rudolph Inc., Kansas City, Mo., USA; 8940 series, made for Cortex Biophysik GmbH, Leipzig, Germany), which was carefully adjusted to the patient's face and checked for air leaks. The patients were able to move freely without discomfort because of the light weight of the equipment $(650 \mathrm{~g})$. The length of the poles (Spin Pole Package; Leki, Buffalo, N.Y., USA) was adjusted for each patient.

During testing, we performed breath-by-breath measurements using a portable system $\left(\right.$ MetaMax $^{\circledR} 3 \mathrm{~B}$ portable CPX system; Cortex Biophysik), which was calibrated before each test. Moreover, an online telemetric assessment of the quality of data collection was done during the testing. The measured variables during exercise testing were: $\dot{\mathrm{VO}}_{2}$, carbon dioxide production, minute ventilation ( $\dot{V} E)$ and heart rate (HR). For both dyspnea and leg discomfort, RPE was assessed immediately after each protocol using a modified Borg scale ranging from 0 to 10 [12].

\section{Statistical Analysis}

Results are presented as means (SD), interquartile ranges or $\mathrm{n}$ (\%) for continuous and categorical variables, respectively. We analyzed the time evolution of the Gaussian continuous variables and compared the four exercise protocols by means of a longitudinal mixed model for repeated measurements taking the intrasubject correlation into account. The characteristics of the slope for the variables $\dot{\mathrm{V}}_{2}, \dot{\mathrm{V}} \mathrm{E}$ and $\mathrm{HR}$ against time for the four exercise protocols were analyzed using a standard regression model. From that model, we assessed whether the slope was within a $\pm 5 \%$ tolerability margin from 0 (i.e. flat slope), in order to evaluate the steadystate profile of each variable. A value of $\mathrm{p}<0.05$ was considered statistically significant.

\section{Results}

One of the 16 COPD patients was excluded because of the patient's inability to tolerate the face mask during the exercise protocols. The characteristics of the 15 patients finally assessed are displayed in table 1 .

Figure 1 depicts the physiological responses throughout each of the four exercise protocols assessed in the study. Of note, the two NW tests and 6MWT showed a $\dot{\mathrm{V}}_{2}$ plateau from minute 3 of the testing. However, $\dot{V} E$ and HR showed a slow incremental profile throughout the 6-min exercise. 
Table 1. Characteristics of the study group $(n=15)$

\begin{tabular}{|c|c|c|}
\hline Male sex, n & $14(93 \%)$ & \\
\hline Current smokers, $\mathrm{n}$ & $2(13 \%)$ & \\
\hline Age, years & $67 \pm 9$ & $(61-74)$ \\
\hline Body mass index, $\mathrm{kg} / \mathrm{m}^{2}$ & $29 \pm 4$ & $(25-31)$ \\
\hline FFMI & $23 \pm 3$ & $(19-25)$ \\
\hline mMRC & $1.2 \pm 0.9$ & $(0.5-2)$ \\
\hline BODE & $1.8 \pm 1.5$ & $(1.0-2.5)$ \\
\hline $\mathrm{FEV}_{1}, \%$ of predicted & $55 \pm 15$ & $(42-64)$ \\
\hline FVC, $\%$ of predicted & $77 \pm 19$ & $(61-85)$ \\
\hline $\mathrm{FEV}_{1} / \mathrm{FVC}$ & $53 \pm 12$ & $(42-61)$ \\
\hline TLC, \% of predicted & $98 \pm 17$ & $(84-104)$ \\
\hline IC, $\%$ of predicted & $78 \pm 19$ & $(66-92)$ \\
\hline $\mathrm{RV}, \%$ of predicted & $142 \pm 27$ & $(128-161)$ \\
\hline $\mathrm{D}_{\mathrm{L}} \mathrm{CO}, \%$ of predicted & $65 \pm 14$ & $(54-77)$ \\
\hline $\mathrm{D}_{\mathrm{L}} \mathrm{CO} / \mathrm{VA}, \%$ predicted & $66 \pm 12$ & $(53-75)$ \\
\hline $\mathrm{PaO}_{2}, \mathrm{~mm} \mathrm{Hg}$ & $76 \pm 10$ & $(67-84)$ \\
\hline $\mathrm{PaCO}_{2}, \mathrm{~mm} \mathrm{Hg}$ & $40 \pm 5$ & $(36-44)$ \\
\hline
\end{tabular}

Data are presented as means $\pm \mathrm{SD}$ (interquartile ranges) or $\mathrm{n}$ (\%) for numerical and categorical variables, respectively. FFMI = Fat-free mass index $\left(\mathrm{kg} / \mathrm{m}^{2}\right) ;$ mMRC $=$ modified Medical Research Council dyspnea scale; BODE = BODE index; $\mathrm{FEV}_{1}=$ forced expiratory volume in the 1 st $\mathrm{s} ; \mathrm{FVC}=$ forced vital capacity; $\mathrm{TLC}=$ total lung capacity; IC = inspiratory capacity; RV = residual volume; $\mathrm{D}_{\mathrm{L}} \mathrm{CO}=$ diffusion capacity of the lung for carbon monoxide; $\mathrm{D}_{\mathrm{L}} \mathrm{CO} / \mathrm{VA}=\mathrm{D}_{\mathrm{L}} \mathrm{CO}$ to alveolar volume ratio; $\mathrm{PaO}_{2}=$ partial $\mathrm{O}_{2}$ pressure in arterial blood; $\mathrm{PaCO}_{2}=$ partial $\mathrm{CO}_{2}$ pressure in arterial blood.

Table 2 displays the physiological measurements expressed as mean values of the last $15 \mathrm{~s}$ of each test. Both $6 \mathrm{mNW}_{\text {ground }}$ and $6 \mathrm{mNW}_{\text {sand }}$ presented similarly higher $\dot{\mathrm{V}} \mathrm{O}_{2}\left(\mathrm{ml} \cdot \mathrm{kg}^{-1} \cdot \mathrm{min}^{-1}\right)$ than the other two exercise protocols. Briefly, $6 \mathrm{mNW}_{\text {ground }} \dot{\mathrm{VO}}_{2}$ was 17 and $11 \%$ higher than the values obtained in 6MWT and SWT, respectively ( $\mathrm{p}<0.05$ each), whereas $6 \mathrm{mNW}_{\text {sand }} \dot{\mathrm{VO}}_{2}$ was 22 and $16 \%$ above the measurements obtained during 6MWT and SWT, respectively ( $\mathrm{p}<0.05$ each). As expected, peak $\dot{\mathrm{V}} \mathrm{O}_{2}\left(\mathrm{ml} \cdot \mathrm{kg}^{-1} \cdot \mathrm{min}^{-1}\right)$ in the SWT was $6 \%$ higher than that observed in the 6MWT $(p<0.05)$. Similar trends were seen for both $\dot{V}$ and HR (table 2). Of note, however, no differences in $\dot{V}$ E were seen between SWT and 6MWT. Moreover, $6 \mathrm{mNW}_{\text {sand }}$ HR was significantly higher compared with the other three exercise protocols $(7,11$ and $13 \%$ for $6 \mathrm{mNW}_{\text {ground }}$, SWT and $6 \mathrm{MWT}$, respectively; $\mathrm{p}<$ 0.05 each) without differences in HR among these three exercise protocols.

No differences in dyspnea rating assessed after $6 \mathrm{mNW}_{\text {ground }}, 6 \mathrm{MWT}$ and SWT were observed (table 2). In contrast, $6 \mathrm{mNW}_{\text {sand }}$ showed significantly higher dyspnea rating compared with the other three exercise pro-

Physiology of Nordic Walking in COPD

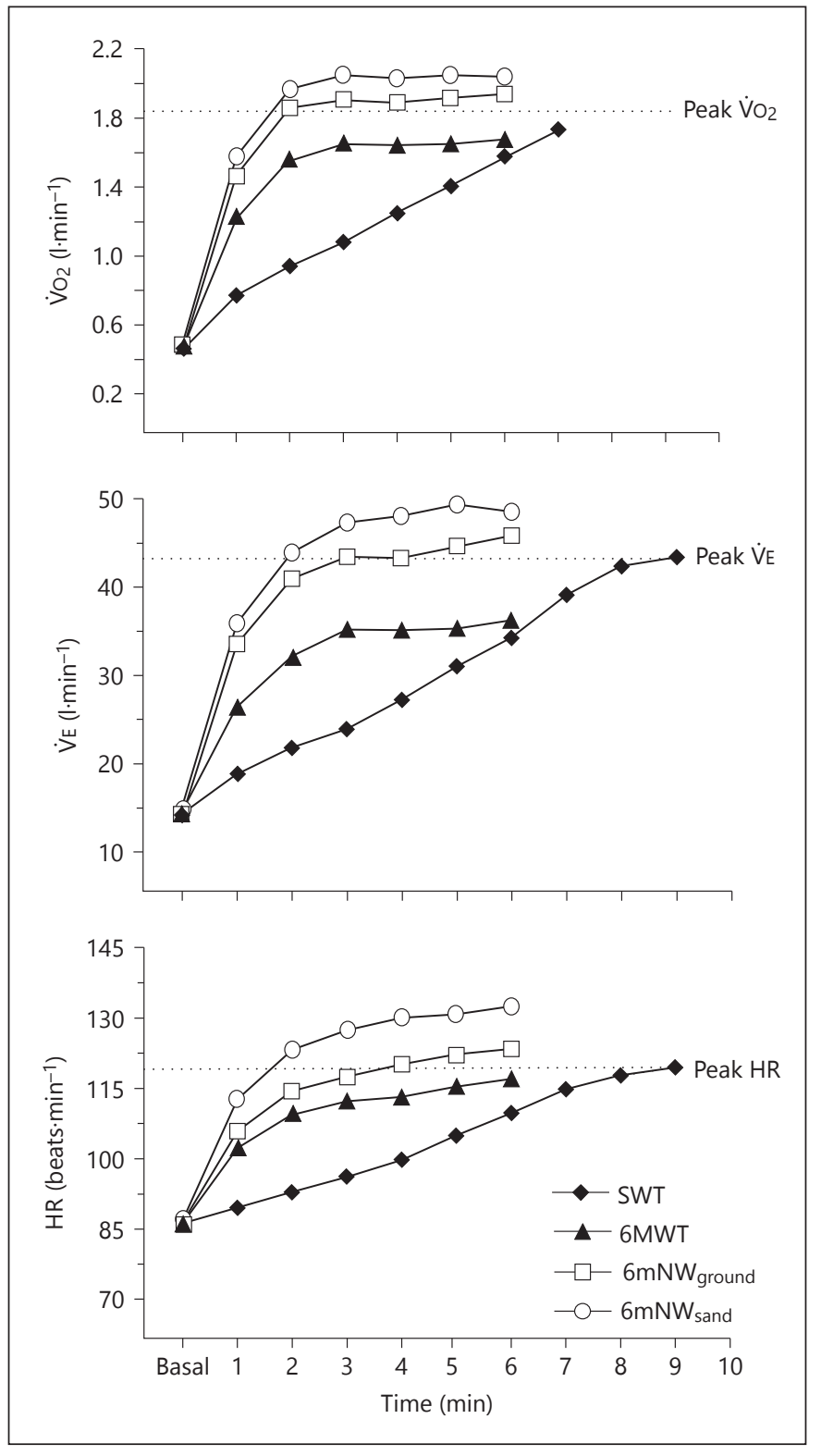

Fig. 1. $\dot{\mathrm{V}}_{2}, \dot{\mathrm{V} E}$ and $\mathrm{HR}$ responses during the four walking protocols.

tocols: 21,24 and $27 \%$ for $6 \mathrm{MWT}, 6 \mathrm{mNW}_{\text {ground }}$ and SWT, respectively ( $\mathrm{p}<0.05$ each). Likewise, leg fatigue rating reported after $6 \mathrm{mNW}_{\text {sand }}$ was also significantly higher than that reported after $6 \mathrm{mNW}_{\text {ground }}(31 \%$; $\mathrm{p}<0.05)$.

\section{Discussion}

\section{Main Findings}

To our knowledge, this is the first study exploring the physiological responses to NW in COPD patients. The 
Table 2. Physiological responses to the different exercise tests

\begin{tabular}{|c|c|c|c|c|c|c|c|c|}
\hline \multirow[b]{2}{*}{ Total distance, $\mathrm{m}$} & \multicolumn{2}{|l|}{ SWT } & \multicolumn{2}{|c|}{ 6MWT } & \multicolumn{2}{|c|}{$6 \mathrm{mNW}_{\text {ground }}$} & \multicolumn{2}{|c|}{$6 \mathrm{mNW}_{\text {sand }}$} \\
\hline & 420 & (98) & 537 & $(65)^{\mathrm{a}}$ & 527 & $(58)^{\mathrm{a}, \mathrm{b}}$ & 375 & $(86)^{a-c}$ \\
\hline$\dot{\mathrm{V}}_{2}, 1 \cdot \mathrm{min}^{-1}$ & 1.82 & $(0.46)$ & 1.68 & $(0.45)$ & 1.94 & $(0.53)^{\mathrm{b}}$ & 2.04 & $(0.53)^{\mathrm{a}, \mathrm{b}}$ \\
\hline$\dot{\mathrm{V}} \mathrm{CO}_{2}, 1 \cdot \mathrm{min}^{-1}$ & 1.46 & $(0.35)$ & 1.32 & $(0.35)^{\mathrm{a}}$ & 1.59 & $(0.42)^{\mathrm{b}}$ & 1.70 & $(0.46)^{a, b}$ \\
\hline$\dot{\mathrm{VE}}, 1 \cdot \mathrm{min}^{-1}$ & 42 & $(10)$ & 36 & $(8)$ & 46 & $(11)^{\mathrm{b}}$ & 49 & $(13)^{\mathrm{a}, \mathrm{b}}$ \\
\hline $\mathrm{HR}$, beats $\cdot \mathrm{min}^{-1}$ & 119 & $(18)$ & 117 & $(18)$ & 123 & $(15)^{\mathrm{b}}$ & 132 & $(15)^{\mathrm{a}-\mathrm{c}}$ \\
\hline Leg discomfort, modified Borg scale & 3.3 & $(1.6)$ & 3.2 & $(1.7)$ & 2.5 & $(2.0)$ & 3.6 & $(2.0)^{\mathrm{c}}$ \\
\hline
\end{tabular}

Data are means (SD) of the last $15 \mathrm{~s}$ in NW tests, and 6MWT and the last $5 \mathrm{~s}$ in the SWT. Dyspnea and leg discomfort ratios were assessed immediately after each test. ${ }^{\mathrm{a}} \mathrm{p}<0.05$ vs. SWT, ${ }^{\mathrm{b}} \mathrm{p}<0.05$ vs. $6 \mathrm{MWT},{ }^{\mathrm{c}} \mathrm{p}<0.05$ vs. $6 \mathrm{mNW}_{\text {ground }}$.

main findings were that: (1) NW $\left(6 \mathrm{mNW}_{\text {ground }}\right)$ results in an increase in $\dot{\mathrm{V}}_{2}$, $\dot{\mathrm{V}}$ and $\mathrm{HR}$ levels compared with standard walking (6MWT); (2) $\dot{\mathrm{VO}}_{2}$ levels achieved during the two NW modalities showed a steady-state profile indicating high but sustainable exercise intensity, and (3) the increase in oxygen uptake generated during $6 \mathrm{mNW}_{\text {ground }}$ does not translate into a higher RPE compared with standard walking $(6 \mathrm{MWT})$ in these patients.

\section{Contributions Beyond the Existing Knowledge}

Previous studies in healthy subjects [4-7] have reported a systematic increment in $\dot{\mathrm{V}}_{2}$ levels during NW compared with standard walking, ranging from 7 to $23 \%$. The present investigation showed an increase of $17 \%$ in $\dot{\mathrm{V}}_{2}$ during $6 \mathrm{mNW}_{\text {ground }}$ compared with $6 \mathrm{MWT}$ and a parallel increase in $\mathrm{VE}$ (28\%). Moreover, the current study clearly indicates that in patients with moderate-to-severe COPD, as previously reported in healthy subjects $[5,6]$, the increase in $\dot{\mathrm{V}}_{2}$ during $6 \mathrm{mNW}_{\text {ground }}$ was not accompanied by a parallel increase in RPE. Even more importantly, the $\dot{\mathrm{VO}}_{2}$ profile during the two NW protocols $\left(6 \mathrm{minNW}_{\text {ground }}\right.$ and $\left.6 \mathrm{minNW}_{\text {sand }}\right)$ indicated sustainability over time of this exercise modality irrespective of the walking surface. The changes in $\dot{\mathrm{VO}}_{2}$ during NW can be reasonably attributed to the increase in exercising muscle mass. $\mathrm{VO}_{2}$ during NW exceeded $\dot{\mathrm{VO}}_{2}$ peak assessed with SWT (table 2). It is well recognized that NW involves recruitment of the upper limb, trunk and lower extremity muscles, whereas standard walking is mostly reduced to lower limb muscle mass.

$6 \mathrm{mNW}_{\text {sand }}$ presented significantly higher both dyspnea and leg discomfort scores than $6 \mathrm{mNW}_{\text {ground, }}$ but $\dot{\mathrm{VE}}$ was similar between the two modalities of NW. We hypothesize that the characteristics of the pattern can be ex- plained by different walking ergonomics due to the surface, i.e. higher surface friction and increased difficulty to keep in balance while walking over soft sand should be expected. The lower $6 \mathrm{mNW}_{\text {sand }}$ walking distance $(-33 \%$; $\mathrm{p}<0.05$ ) compared with $6 \mathrm{mNW}_{\text {ground }}($ table 2) supports this interpretation.

\section{Clinical Applicability of the Results}

One of the central challenges of pulmonary rehabilitation programs for COPD patients [13] is to provide longterm cost-effective strategies aiming to enhance daily physical activity of these patients [14]. Therefore, NW has been reported to be an effective method to improve both aerobic capacity and physical activity in patients with COPD [3]. The current study reinforces the message by providing novel information on the physiological responses to NW in COPD patients.

We would like to stress the potential of NW as an exercise training method for COPD patients in urban environments, highlighting that NW generates higher intensities than standard walking, but the cardiorespiratory challenge is still sustainable and can be applied to train skeletal muscles. Consequently, we may obtain a higher training stimulus with similar perceived effort. Moreover, for the practice of NW, urban environments provide both an easily accessible and attractive setting as a low-tech, low-cost training approach that may result in enhanced long-term adherence to the program.

\section{Strengths and Limitations}

The current results may show limitations for generalization of the main message to very severe COPD patients that were not included in the study because of their inability to support the face mask and, in some 
cases, the need of long-term oxygen therapy. However, while acknowledging this fact, we would like to stress that patients with moderate/severe disease are the target for this kind of community-based preventive interventions with the aim to improve their health status and, ultimately, to delay disease progression. Finally, the reliability of the results is based on the study design and the quality control procedures, including equipment calibration and telemetric data collection and control during the tests.

\section{Conclusions}

The results of the study suggest that NW could be an appropriate walking modality to train patients with moderate to severe COPD because it generates higher exercise intensity at the same RPE compared with standard walking. However, further studies comparing the impact of
NW and standard walking on health outcomes in COPD patients with different degrees of COPD severity are needed before NW is included into community-based rehabilitation programs.

\section{Acknowledgments}

The authors would like to thank Ferran Torres [Institute of Biomedical Research August Pi i Sunyer (IDIBAPS), Biostatistics and Data Management Platform, Barcelona] for his support in statistical analyses. We also acknowledge the support received from the staff of the Respiratory Diagnostic Center of the Hospital Clínic de Barcelona.

The study was financially supported by NEXES-Supporting Healthier and Independent Living for Chronic Patients and Elderly (UE grant CIP-ICT-PSP-2007-225025); grants from the Fondo de Investigación Sanitaria (FIS PI-061510); Col-legi de Fisioterapeutes de Catalunya R02-2007 and the 2nd Solidary Triathlon organized by the Barcelona city council sports center Can Ricart.

\section{References}

1 International Nordic Walking Federation. http://inwa-nordicwalking.com/.

- 2 Tschentscher M, Niederseer D, Niebauer J: Health benefits of Nordic walking: a systematic review. Am J Prev Med 2013;44:76-84.

3 Breyer MK, Breyer-Kohansal R, Funk GC, Dornhofer N, Spruit MA, Wouters EF, Burghuber OC, Harti S: Nordic walking improves daily physical activities in COPD: a randomized controlled trial. Respir Res 2010;11:112.

-4 Schiffer T, Knicker A, Hoffman U, Harwig B, Hollmann, Struder HK: Physiological responses to Nordic walking, walking and jogging. Eur J Appl Physiol 2006;98:56-61.

5 Church TS, Earnest CP, Morss GM: Field testing of physiological responses associated with Nordic walking. Res Q Exerc Sport 2002;73: 296-300.

6 Rodgers CD, VanHeest JL, Schachter CL: Energy expenditure during submaximal walking with Exerstriders. Med Sci Sports Exerc 1995; 27:607-611.

7 Porcari JP, Hendrickson TL, Walter PR, Terry L, Walsko G: The physiological responses to walking with and without Power Poles on treadmill exercise. Res Q Exerc Sport 1997;68: 161-166.
8 Lahaije A, van Helvoort H, Dekhuijzen R, Heijdra Y: Can COPD patients who hyperinflate during daily life activities be identified by laboratory tests? Respiration 2013;86:237242.

-9 Vestbo J, Hurd SS, Agustí AG, Jones PW, Vogelmeier C, Anzueto A, Barnes PJ, Fabbri LM, Martinez FJ, Nishimura M, Stockley RA, Sin DD, Rodriguez-Roisin R: Global strategy for the diagnosis, management and prevention of chronic obstructive pulmonary disease: GOLD executive summary. Am J Respir Crit Care Med 2013;187:347-365.

10 Singh SJ, Morgan MD, Scott S, Walters D, Hardman AE: Development of a shuttle walking test of disability in patients with chronic airways obstruction. Thorax 1992;47:10191024.

11 ATS Committee on Proficiency Standards for Clinical Pulmonary Function Laboratories: ATS statement: guidelines for the six-minute walk test. Am J Respir Crit Care Med 2002; 166:111-117.
12 Ries AL: Minimally clinically important difference for the UCSD Shortness of Breath Questionnaire, Borg Scale and Visual Analog Scale. COPD 2005;2:105-110.

-13 Spruit MA, Singh SJ, Garvey C, ZuWallack R, Nici L, Rochester C, Hill K, Holland AE, Lareau SC, Man WD, Pitta F, Sewell L, Raskin J, Bourbeau J, Crouch R, Franssen FM, Casaburi R, Vercoulen JH, Vogiatzis I, Gosselink R, Clini EM, Effing TW, Maltais F, van der Palen J, Troosters T, Janssen DJ, Collins E, GarciaAymerich J, Brooks D, Fahy BF, Puhan MA, Hoogendoorn M, Garrod R, Schols AM, Carlin B, Benzo R, Meek P, Morgan M, Rutten-van Mölken MP, Ries AL, Make B, Goldstein RS, Dowson CA, Brozek JL, Donner CF, Wouters EF, ATS/ERS Task Force on Pulmonary Rehabilitation: An official American Thoracic Society/European Respiratory Society statement: key concepts and advances in pulmonary rehabilitation. Am J Respir Crit Care Med 2013; 188:e13-e64.

14 Hartman JE, Boezen HM, Zuidema MJ, de Greef MH, Ten Hacken NH: Physical activity recommendations in patients with chronic obstructive pulmonary disease. Respiration 2014;88:92-100. 